\section{Capsule endoscopy as a diagnostic aid in obscure gastrointestinal bleed in a child}

Blue Rubber Bleb Nevus Syndrome (BRBNS) is a rare disorder which manifests with a combination of cutaneous and gastrointestinal venous malformations. ${ }^{1}$ The diagnosis is based on the presence of characteristic lesions which look like blue rubber blebs. Gastrointestinal (GI) lesions often bleed spontaneously and may present with either an overt bleed (as in our case) or with occult blood loss and consequent chronic anemia alone. Upper gastrointestinal endoscopy and colonoscopy are commonly used to localize the GI lesions. In cases of obscure gastrointestinal bleed (as in our case), capsule endoscopy provides valuable information about the presence and extent of the lesions, thus facilitating the choice of an appropriate treatment strategy. ${ }^{2}$ There is no pediatric case report in literature till date where capsule endoscopy clinched the diagnosis of BRBNS and aided in the management.

\section{Case Report}

A 9-year old girl presented with a history of melena and sudden pallor. She had had eight similar episodes in the past from the age of one year to the present. The episodes occurred at varying intervals and required blood

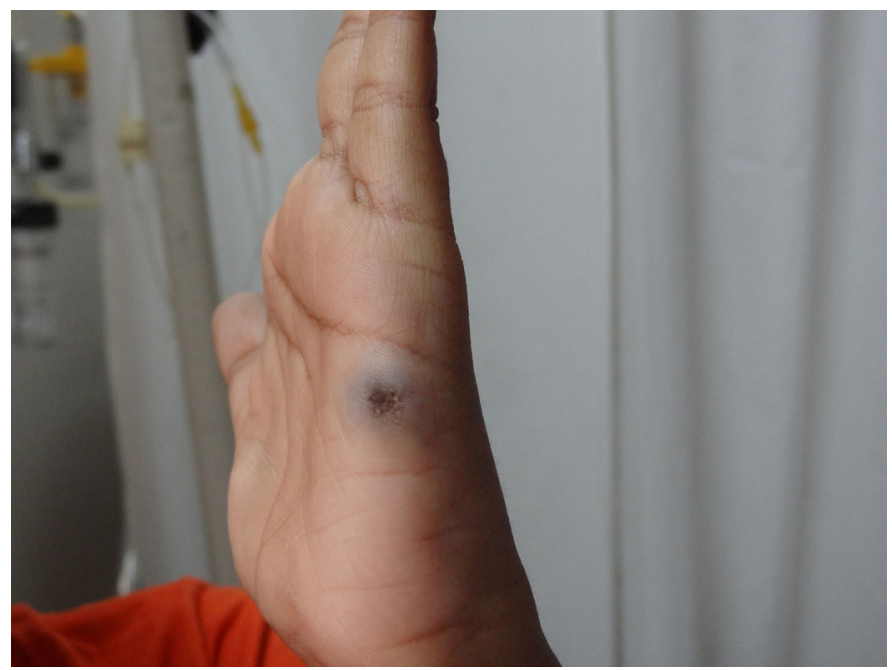

Figure 1: Image showing a bluish cutaneous swelling on the left hand. transfusion every time. She was evaluated elsewhere with upper gastrointestinal endoscopy and colonoscopy, which were both normal. On clinical examination, she had pallor and tachycardia with a normal blood pressure. She also had a bluish coloured swelling along the ulnar border of her left hand and over her left scapula, which was soft, globular, compressible, non-tender, non-pulsatile and immobile with normal skinit over (Figure1) suggestive of a cutaneous blue rubber nevus. Her hemoglobin level at admission was $6 \mathrm{gm} \%$. On peripheral blood smear, the red blood cells were microcytic and hypochromic.Her platelet count and coagulation parameters were within normal limits. A repeat upper gastrointestinal endoscopy and colonoscopy did not show any blue rubber bleb nevi. Since the bleeding source was not readily identifiable by routine esophagogastroduodenoscopy and colonoscopy, this was labelled a case of obscure overt gastrointestinal bleed. She subsequently underwent capsule endoscopy to localize the source of bleed. Capsule endoscopy revealed two blue rubber lesions in the proximal and middle portions of the jejunum (Figure 2). Upon detection of both gastrointestinal and cutaneous lesions, a diagnosis of blue rubber bleb nevus syndrome was confirmed. In view of her frequent transfusion requirements and the lesions

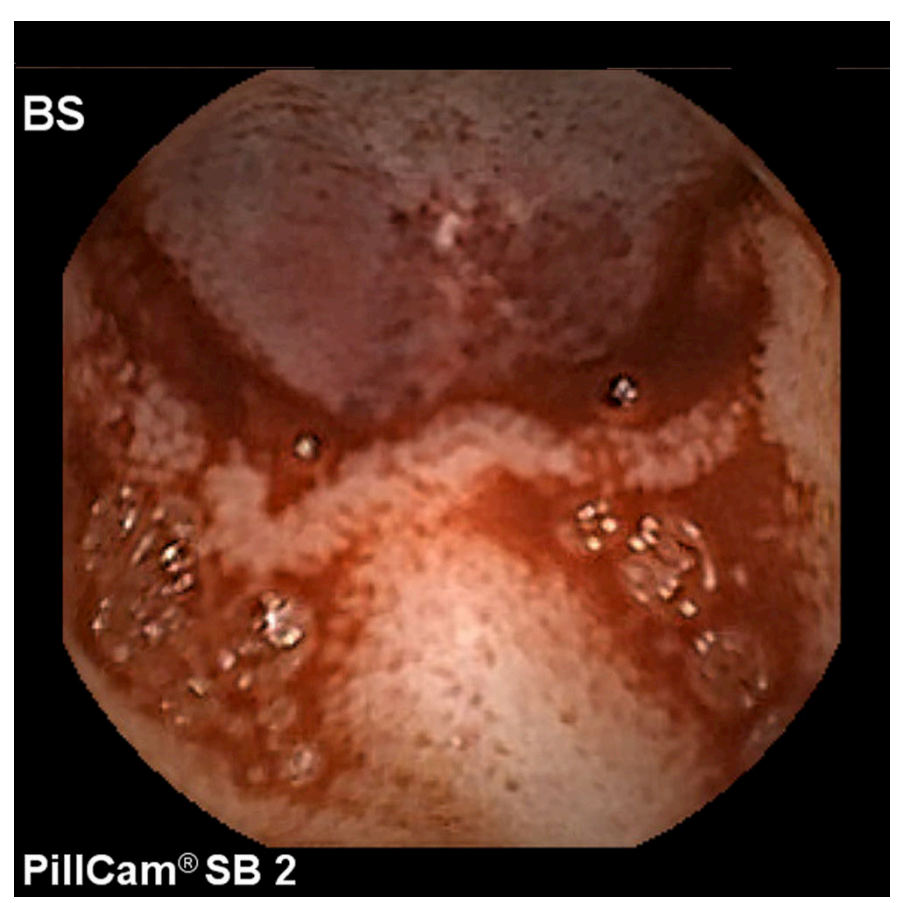

Figure 2: Capsule endoscopy image of the blue rubber bleb nevus with active bleed. 
being restricted to the jejunum, surgery was planned. Subsequently an intraoperative endoscopy confirmed two blue rubber bleb lesions at $25 \mathrm{~cm}$ and $50 \mathrm{~cm}$ from the duodeno-jejunal flexure. She underwent resection and anastomosis of the affected jejunal segment.Two year postoperatively, she is doing well. On periodic follow up visits, she neither had occult blood in her stools nor anemia.

\section{Discussion}

In 1958, William Bennet Bean coined the term Blue Rubber Bleb Nevus Syndrome to describe a rare condition characterized by multiple venous malformations involving the skin and internal organs as, hence it is also called as Bean syndrome. ${ }^{1}$ The venous malformations in BRBNS are often present at birth or in early childhood. Few cases have been described with an autosomal dominant pattern of inheritance, but most cases are sporadic. It is common for the GI lesions to bleed spontaneously and such bleeding may be occult or overt as hematemesis, melena, or rectal bleeding. Small intestine is the most frequently involved extracutaneous organ. To plan management it's crucial to know the distribution of lesions which is unique to each individual.

Obscure gastrointestinal bleeding is defined as bleeding from a difficult to identify source by endoscopy or colonoscopy. Capsule endoscopy, a noninvasive technology is becoming an important method for the visualization of the small bowel in cases with obscure GI bleed. ${ }^{3}$ The capsule is either swallowed by the child or placed endoscopically and tiny wireless camera in the capsule records pictures of digestive tract as it passes through. It provides good visualization of gastrointestinal mucosa.

In previous case reports on BRBNS, upper GI endoscopy and colonoscopy had already shown blue rubber lesions, and capsule endoscopy was done to know small bowel involvement. ${ }^{4,5}$ But in our case, capsule endoscopy alone could demonstrate jejunal lesions and thus helped in making diagnosis of BRBNS. It also helped in determining the extent of involvement of the small bowel and thus plan further management.

The treatment of GI lesions of BRBNS depends on their number, location, size and symptoms. ${ }^{5}$ When the venous malformation is confined to a segment of the GI tract, resection of the involved segment of the gut may be indicated. However, a conservative approach is usually recommended whenever the clinical features and bleeding episodes are mild. ${ }^{6}$ In milder cases, therapy is based on iron supplementation and/or blood transfusion. A range of pharmacological treatments like corticosteroids, interferon, vincristine, octreotide, sirolimus have been described in case reports but sustained response is not well documented. ${ }^{7,8}$ Our patient presented a localized disease with frequent blood transfusion requirement (once/year), so we took a surgical approach and resected the involved segments with favorable result.

With advent and application of new diagnostic modalities like capsule endoscopy, diagnosis and effective treatment can be offered to children with obscure GI bleed, which hitherto remained an enigma. This case highlights the blend of good clinical examination and advanced technology in the form of capsule endoscopy that led to diagnosis and treatment of BRBNS, a rare condition.

JAYA AGARWAL MAYANK MEHROTRA AJMAL HASAN Regency Health Care, Kanpur, India Correspondence: Dr Jaya Agarwal Email:drjaya.agarwal@gmail.com

\section{References}

1. Jennings M, Ward P, Mattocks JL. Blue rubber bleb naevus disease: an uncommon cause of gastro-intestinal tract bleeding. Gut.1988;29:1408-12.

2. Guilhon de Araujo Sant'Anna AM, Dubois J, Miron MC, Seidman EG. Wireless capsule endoscopy for obscure small-bowel disorders: final results of the first pediatric controlled trial. Clin Gastroenterol Hepatol. 2005; 3:26470.

3. Raju GS, Gerson L, Das A, Lewis B; American Gastroenterological Association. American Gastroenterological Association (AGA) Institute medical position statement on obscure gastrointestinal bleeding. Gastroenterology. 2007;133(5):1694-6. 
4. Badran AM, Vahedi K, Berrebi D, Catana D, De Lagausie P, Drouet L et al. Pediatric ampullar and small bowel blue rubber bleb nevus syndrome diagnosed by wireless capsule endoscopy. J Pediatr Gastroenterol Nutr. 2007; 44: 283-6.

5. De Bona M, Bellumat A, De Boni M. Capsule endoscopy for the diagnosis and follow-up of blue rubber bleb nevus syndrome. Dig Liver Dis. 2005; 37: 451-3.

6. Agnese M, Cipolletta L, Bianco MA, Quitadamo P, Miele E, StaianoA.Blue rubber bleb nevus syndrome.ActaPaediatr. 2010;99:632-5.

7. Yuksekkaya H, Ozbek O, Keser M, Toy H. Blue rubber bleb nevus syndrome: successful treatment with sirolimus. Pediatrics. 2012;129:e1080-e1084.

8. Gonzalez D, Elizondo BJ, Haslag S, Buchanan G, Burdick JS, Guzzetta PC et al.Chronic subcutaneous octreotide decreases gastrointestinal blood loss in bluerubber-bleb nevus syndrome.JPediatr Gastroenterol Nutr. 2001; $33: 183-8$.

\section{Synchronous cholangiocarcinoma and gall bladder carcinoma}

The simultaneous occurrence of malignancies in the bile duct and the gall bladder is not frequently reported. They might be arising as a part of the spectrum of rare synchronous malignancies or could be occurring as a result of local spread or metastasis. When considering a diagnosis of synchronous primaries, the criteria that have been used to support this relatively rare occurrence must be taken into account since the two sites are anatomically close to each other and have embryologic similarities as well. Gertsch et al have attempted to describe the criteria to differentiate between the two entities. ${ }^{1}$ The diagnosis of these synchronous tumours is rarely made preoperatively. They should be suspected and looked for in every instance by intraoperative endoscopy and meticulous histologic examination of the gall bladder as they are associated with fairly good prognosis if complete surgical resection is possible. Here, we report a rare case of synchronous cholangiocarcinoma and gall bladder carcinoma in a 35 year old female.

\section{Case Report}

A 35-year old female presented to the OPD with pain abdomen for the past 4 months. Her blood work-up revealed raised values of bilirubin (total bilirubin-7.9 $\mathrm{mg} / \mathrm{dl})$ and alkaline phosphate (720 IU/L). Computed Tomography showed a mass measuring $2.7 \mathrm{~cm} \times 2.5 \mathrm{~cm}$ at the lower end of the common bile duct with cholelithiasis. The patient was taken for surgery, wherein a growth was found in the lower part of the common bile duct with dense adhesions over Calot's triangle, the porta hepatis and the gall bladder. An extended cholecystectomy with extrahepatic common bile duct excision and pancreaticoduodenectomy with a radical lymphadenectomy was performed.

On opening the common bile duct, gross examination revealed a grey-white growth measuring $1.2 \mathrm{~cm} x 1 \mathrm{~cm} \times 1 \mathrm{~cm}$ was present at the resected end encircling it. Cut section of the gall bladder showed wall thickening in the fundus region measuring $1 \mathrm{~cm}$.

Histological examination showed a moderately differentiated adenocarcinoma of the gall bladder (Figure 1) and the common bile duct (Figure 3) with extension beyond the serosa and focally involving the pancreas and the muscle layer of the duodenum. The adjacent mucosa of both showed in situ changes (Figure 2) without any direct continuity (Figure 4) between the two tumors. Solitary lymph node metastasis was also present. Immunohistochemistry was done wherein both the gall bladder carcinoma and the cholangiocarcinoma showed more than 10 per cent Ki67 positivity and absence of $\mathrm{p} 53$ mutation.

\section{Discussion}

Cholangiocarcinoma is an uncommon tumour seen mainly in the elderly. The synchronous occurrence of 